356 Jorgensen: Ueber den sogenannten Herapathit

Ueber den sogenaunten Herapathit und ähnliche Acidperjodide;

ron

S. M. Jörgensen.

(Fortsetzung.) ${ }^{1}$ )

D. Chinidinverbindungen.

Bei Chinidin wird hier das Chinidin von Pasteur, das Conchinin von Hesse verstanden. Das zu nachstehenden Untersuchungen verwendete Alkaloid wurde zum grossen Theil aus dem charakteristischen, schwer löslichen, jodwasserstoffsauren Salze dargestellt.

$$
\begin{aligned}
& \text { 1tes Chinidinperjodidsulfat. } \\
& 2 \mathrm{C}_{20} \mathrm{H}_{24} \mathrm{~N}_{2} \mathrm{O}_{2}, \mathrm{SH}_{2} \mathrm{O}_{4}, 2 \mathrm{HJ}, \mathrm{J}_{4} \text {. }
\end{aligned}
$$

Diese Verbindung warde von Herapath dargestellt und von ihm und Muspratt mit dem bereits angegebenen $\left.{ }^{2}\right)$ Resultat analysirt. Sie wird leicht und sicher erhalten beim Auflösen des Chinidins in der theoretischen Menge normaler Schwefelsäure und heissem Weingeist und Zusatz der berechneten Menge Jodwasserstoffäüure und etwas weniger Jod in heisser weingeistiger Lösung, als die Formel verlangt. Beim Erkalten krystallisirt die Verbindung und ist nach einer Umkrystallisation rein. Sie bildet centimeterlange, granatrothe Nadeln von bläulichem Glanz. Sie absorbiren kaum polarisirtes Licht, sind stark glänzend, häufig aber gestreift und unregelmäвsig ausgebildet. Bei schnellem Erkalten erhält man jedoch oftmals dünnere Blätter, von $\infty \overline{\mathrm{P}} \infty$ und mehreren makrodiagonalen Atomen begrenzt. Ausser $\overline{\mathrm{P}} \infty$ (gef. in 6 Messungen an 4 Krystallen $\overline{\mathrm{P}} \infty: \infty \overline{\mathrm{P}} \infty=119,5^{\circ}$ bis $120^{\circ}$ ) beobachtete ich $3 / 2 \overline{\mathrm{P}} \infty, 2 / 3 \overline{\mathrm{P}} \infty$ und $1 / 3 \overline{\mathrm{P}} \infty$, bisweilen alle auf dem-

1) Fortsetzung der S. 268 abgebrochenen Abhandlung.

2) S. 218 . 
selben Krystall, dessen Enden dann abgerundet erschienen. Aus $\overline{\mathrm{P}} \infty: \infty \overline{\mathrm{P}} \infty=120^{\circ}$ berechnen sich:

$3 / 2 \overline{\mathrm{P}} \infty: \infty \overline{\mathrm{P}}_{\infty}=130,9^{\circ}$. Gef. im Durchsohnitt 131,50

$$
\begin{aligned}
& 2 / 3 \overline{\mathrm{P}} \infty: \infty \overline{\mathrm{P}} \infty=111^{\circ} \text {. " " " } \\
& 1 / 8 \overline{\mathrm{P}} \infty: \infty \overline{\mathrm{P}} \infty=100,1^{0} \text {. } \quad " \quad, \quad, \quad 101,5^{\circ}
\end{aligned}
$$

Das auf $100^{\circ}$ erhitzt gewesene Salz decrepitirt stark beim Erkalten, was Herapath auch bemerkt, so dass Theile der Krystalle weit herumspringen. Schon dies deutet an, dass die Verbindung wasserfrei ist. Auch verliert die neben Vitriolöl getrocknete Verbindung bei $100^{\circ}$ im Durchschnitt nur 0,4 Proc. Wasser (von 0,36 bis 0,45 Proc.), was bei den Analysen in Abrechnung gebracht ist.

Die Analysen ergaben:

\begin{tabular}{lrrrrrrr} 
& & \multicolumn{4}{c}{ Gefunden } & \multicolumn{3}{c}{ Herap. Muspr. } \\
& Rechn. & $\mathbf{4}$ & \multicolumn{2}{c}{ a. } & b. & c. & \multicolumn{2}{c}{ fanden im } & Durohschn \\
$\mathbf{C}$ & $\mathbf{3 1 , 7 9}$ & $\mathbf{3 1 , 3 6}$ & - & $\mathbf{3 1 , 5 6}$ & - & 32,76 & $\mathbf{3 2 , 1 5}$ \\
$\mathbf{H}$ & $\mathbf{8 , 4 4}$ & $\mathbf{3 , 5 8}$ & - & $\mathbf{3 , 6 1}$ & - & $\mathbf{3 , 9 8}$ & $\mathbf{3 , 9 7}$ \\
$\mathbf{N}$ & $\mathbf{8 , 7 1}$ & - & - & - & - & 4,44 & - \\
$\mathbf{S O}$ & $\mathbf{5 , 3 0}$ & $\mathbf{5 , 3 4}$ & - & $\mathbf{5 , 4 3}$ & - & $\mathbf{6 , 3 4}$ & $\mathbf{6 , 2 6}$ \\
$\mathbf{J}$ & $\mathbf{5 0 , 4 6}$ & $\mathbf{5 0 , 0 6}$ & $\mathbf{5 0 , 0 7}$ & $\mathbf{5 0 , 0 6}$ & $\mathbf{5 0 , 1 3}$ & $\mathbf{3 9 , 7 3}$ & $\mathbf{3 9 , 7 8}$ \\
Froieres Jod & $\mathbf{3 3 , 6 5}$ & $\mathbf{3 3 , 7 0}$ & - & - & - & - & -
\end{tabular}

Verbrauchtes Thalliumjodür auf 1 Mol.: gef. 1,97 Mol., Rechn. 2.

Rationelle Formel daher:

$$
\mathrm{J}_{2} \text {.J. . HCh H. O. } \mathrm{SO}_{2} \text {. O . } \mathrm{HChH} \text {.J. } \mathrm{J}_{2} \text {. }
$$

Es lag mit Rücksicht auf Herapatb's und Muspratt's Jodbestimmungen nahe, anzunehmen, dass sie und ich ganz verschiedene Verbindungen untersuchten. Hiergegen spricht aber, dass die von Herapath angegebenen physikalischen Eigenschaften (u. a. auch das eigenthümliche Decrepitationsphänomen) ganz meinen Beobachtungen entsprechen, so wie, was ich oben ${ }^{1}$ ) bemerkte. Ausserdem kann ich hier hervorheben, dass man selbst aus den theoretischen Mengen Chinidin, Schwefelsäure und Jodwasserstoff, aber

1) S. 223 . 
nur der Hälfte der berechneten Jodmenge, noch immer dieselbe Verbindung erbält (s. die dritte obiger Analysen). Auch kann ich binzufügen, dass mehrere Versuche, das Jod in dieser Verbindung durch Kochen desselben in feingepulvertem Zustande mit wässrigem Silbernitrat and schliesslich wit verdünnter Salpetersäure, folglich unter weit günstigeren Verhältnissen als denen, unter welchen Herapath und (wahrscheinlicher Weise) Muspratt gearbeitet haben, zu bestimmen, mir allzu niedrige Resultate (44 bis 46 Proc. Jod) gaben. Dass das Jodsilber in obigen Analysen rein war, geht daraus hervor, dass es in den zwei Analysen nach der (S. 221) beschriebenen Weise zwei Mal gefällt wurde. Dass die von mir analysirte Verbindung keine Spur freien Jods enthielt, wurde dadurch angezeigt, dass sie Aether, Chloroform, Schwefelkohlenstoff auch nicht die geringste Färbung mittheilte, so wie dadurch, dass die heisse weingeistige Lösung, mit Quecksilber geschüttelt, keine Spur Quecksilberjoduir bildete. Dagegen bildet sich hier ein Quecksilberjodiddoppelsalz, in heissem Weingeist löslich (bei nicht hinreichendem Weingeist schmilzt es beim Erwärmen za. gelben Tropfen), daraus sich bei langsamem Erkalten als Rosetten ziemlich grosser, rectangulärer; stark gestreifter Prismen abscheidend. Zur Darstellung des unter c untersuchten Präparats wurde das umkrystallisirte Acidperjodid selbst in Wasser vertheilt, durch Schwefelwasserstoff zersetzt, das durch Stehon von Schwefelwasserstoff befreite Filtrat durch Ammoniak gefällt, das gewaschene Alkaloid in verdünnter Salzsäure gelöst, die Lösung mit Ammoniak neutralisirt und mit Jodkalium gefällt. Das so gewonnene umkrystallisirte Jodid, das, bei $100^{\circ}$ getrocknet, 28,0 Proc. Jod (Rechn. 28,09) hält, wurde endlich in obiges Acidperjodid verwandelt. Auch eine mit letzterem in Eigenschaften ganz analoge, anten zu beschreibende Selensäureverbindung hat entsprechende Zusammensetzung. 
2 tes Chinidinperjodidsulfat. $3 \mathrm{C}_{20} \mathrm{H}_{24} \mathrm{~N}_{2} \mathrm{O}_{2}, 3 \mathrm{SH}_{2} \mathrm{O}_{4}, 2 \mathrm{HJ}, \mathrm{J}_{8}$.

Schon oben ${ }^{1}$ ) wurde bemerkt, dass Herapath ein optisch wirksames Chinidinsalz dieser Gruppe oberfä̈chlich untersucht hat. Er leitet seine Mittheilung mit der Bemerkung ein, dass die conc. Lösung des sauren schwefelsauren Chinidins beim Sieden grünlich wird, was nach ihm eine Veränderung in dem Chinidinmolekül andeutet, und da er unmittelbar darauf mittheilt, dass das optisch wirksame Salz aus solchen und nur aus solchen Lösungen entsteht, während verdünnte Ijösungen immer die oben als 1tes Chinidinperjodidsulfat beschriebene Verbindung liefern, während nie beide Salze aus derselben Lösung abscheiden, so scheint es seine Meinung zu sein, dass das optische Salz eine andere Chinidinmodification als die gewöhnliche enthält. Dem ist aber nicht so, wenigstens wenn die jetzt zu beschreibende Verbindung, welche in allen physikalisehen Eigenschaften mit der von Herapath beschriebenen übereinstimmt, wirklich mit letzterer identisch ist. Eine Analyse von Herapath's Verbindung liegt nicht vor. Die Bildungsbedingungen meiner Verbindung sind, dass die Flüssigkeit sehr überschüssige Schwefelsäure, aber sehr wenig Jodwasserstoffsäure hält, dass sie weit weniger als die theoretische Jodmenge hält, und dass eine Temperatur von etwa $60^{\circ}$ eingehalten wird. War die Flüssigkeit nicht hinreichend sauer oder zu reich an Jodwasserstoff, so wird zugleich oder allein 1 tes Chinidinperjodidsulfat gebildet, hält sie zu viel Jod, oder war die Temperatur zu hoch, so wird zwar das Salz erhalten, aber gewöhnlich mit theerähnlichen Produkten gemischt, von welchen es nicht getrennt werden kann. Die Wirkung von zu viel Jodwassersı̂foff ist so gross, dass ich .ganz aufgeben musste, diese Säure direct zuzusetzen, die gekocht gewesene Jodlösung enthält hinreichend. Von den vielen verschiedenen Gemischen, die ioh unter ungleichen Bedingungen versuchte, empfehle ich: 3,5 Grm. Chinidin (von 8 bis 10 Proc. Wassergehalt),

1) S. 219. 
360 Jörgensen: Ueber den sogenannten Herapathit

$25 \mathrm{Ccm}$. verdünnter Schwefelsäure (17 Proc. $\mathrm{SO}_{3}$ haltend) und $60 \mathrm{Ccm}$. Weingeist von 92 Proc. T., hierzu werden $2 \mathrm{Grm}$. Jod in $60 \mathrm{Ccm}$. Weingeist von $92 \%$ T. gesetzt. Die Jodlösung darf keine höhere Temperatur als $50^{\circ}$ haben, und viel höher darf auch nicht die Temperatur der sauren Flüssigkeit sein. Beim Umrühren füngt das Gexnisch fast augenblicklich an, Krystalle abzuscheiden, und bei langsamem Erkalten und Stehen vergrössert sich die Menge derselben sehr beträchtlich, obwohl man auch nicht annähernd die theoretische Menge erreicht, u. A., weil in so saurer Flüssigkeit gearbeitet werden muss. Die Verbindung wird mit kaltem, verdünntem Weingeist gewaschen und neben Vitriolöl getrocknet.

In Masse bildet sie olivenbraune (in der Flüssigkeit suspendirt metallisch glänzende) rhombische Blätter oder kleine, abgeplattete Prismen. Selten oder nie ist $\overline{\mathbf{P}} \infty$ mit mehr als einer Fläche an jedem Ende des Prisma ausgebildet, so dass letzteres als Rhombus oder Parallelogramm von 111,330 (Durchschnitt von 18 Messungen an $5 \mathrm{Kry}$ stallen) erscheint. Die optische Wirkung ist sehr hervortretend: I| der einen Seite des Rhombus oder der Längsaxe des Prisma ganz hell olivengelb; + undurchsichtig.

Die Analyse der neben Vitriolöl getrockneten Verbindung, welche unter dem Mikroskop ganz frei von dem vorhergehenden Salze erschien, ergab:

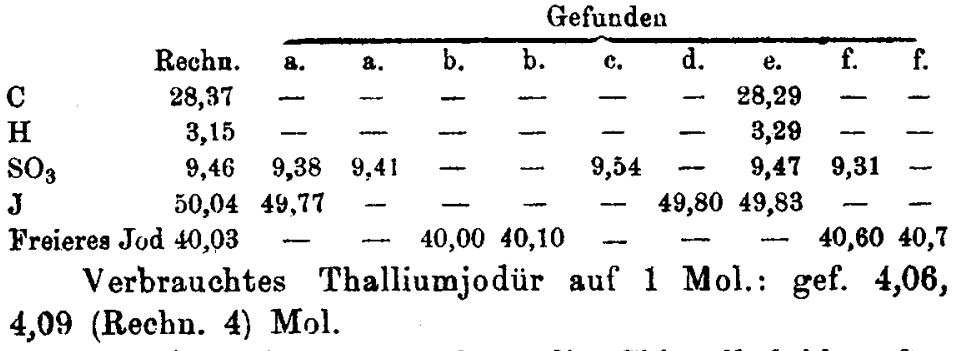

Berticksichtigt man, dass die Chinaalkaloide öfter übersaure Salze bilden, z. B. $\mathrm{C}_{20} \mathrm{H}_{24} \mathrm{~N}_{2} \mathrm{O}_{2}, 2 \mathrm{SH}_{2} \mathrm{O}_{4}$, in welchen das mit 4 At. Wasserstoff verbundene Alkaloid vierwerthig auftritt, so erscheint folgende Formel der Verbindung einigermaassen wahrscheinlich: 


$$
\begin{aligned}
& \mathrm{J}_{4} \cdot \mathrm{J} . \mathrm{H} \mathrm{Ch} \mathrm{H} \cdot \mathrm{O} . \mathrm{SO}_{2} \cdot \mathrm{O} \cdot \mathrm{H} \\
& \mathrm{J}_{4} \text {. J . H Ch H.O.SO } \mathrm{H} . \mathrm{O} \cdot \mathrm{SO}_{2} . \mathrm{O} . \mathrm{H} \\
& \mathrm{H} . \mathrm{O} .
\end{aligned}
$$

Beim Aufbewahren scheint die Verbindung eine eigenthümliche Veränderung zu erleiden, wobei das in freierem Zustande vorhandene Jod allmählich verringert wird, obne dass die totale Jodmenge merklich vermindert wird. In einem Präparat, das länger als ein Jahr verschlossen gestanden hatte, war so die Menge des freieren Jods bis auf 35,5 Proc. herabgesunken, während die frisch dargestellte Verbindung immer sehr nahe 40 Proc. hält. Dies eigenthümliche Verhalten machte eine oftmals wiederholte Darstellung und Analyse dieser Verbindung nöthig, um so mehr, als eine andere Formel, die aber nur 38,02 Proc. freieres Jod verlangt, fast eben so gut wie die obige den gefundenen Zahlen entspricht, nämlich:

$$
\begin{array}{r}
4 \mathrm{C}_{20} \mathrm{H}_{24} \mathrm{~N}_{2} \mathrm{O}_{2}, 4 \mathrm{SH}_{2} \mathrm{O}_{4}, 3 \mathrm{HJ}, \mathrm{J}_{10} \text {. } \\
\text { Rechn. }
\end{array}
$$

$\begin{array}{lrr}\mathrm{C} & \mathbf{2 8 , 7 3} & \mathbf{2 8 , 2 9} \\ \mathrm{H} & \mathbf{3 , 2 0} & \mathbf{3 , 2 9} \\ \mathrm{SO}_{3} & \mathbf{9 , 5 7} & \mathbf{8 , 4 5} \\ \mathrm{J} & \mathbf{4 9 , 4 0} & \mathbf{4 9 , 8 0}\end{array}$

Letztere Formel schien um so wahrscheinlicher, als eine sehr ähnliche Selensäureverbindung, welche ausserdem mit der Schwefelsäureverbindung isomorph zu sein schien, Zahlen geliefert hat, welche kaum mit einer anderen Formel als der mit der letzteren Formel analogen sich vereinigen lässt. Nichts desto weniger glaube ich nicht, dass die letztgenannte Formel die richtige ist, und zwar aus folgenden Gründen. Die Jodbestimmungen fallen gewöhnlich ein wenig zu niedrig, die Schwefelsäurebestimmungen ein wenig zu hoch aus; mit der letzteren Formel sollte das Umgekehrte der Fall sein. In der frisch dargestellten Verbindung habe ich immer etwa 2 Proc. mehr freieres Jod gefunden, als letztere Formel verlangt, und ein solcher Fehler scheint mir bei den angewandten Methoden kaum möglich. Endlich ist die obenerwähnte Isormorphie eigenthümlicher Art. Bei dem Seleniat kommen Blätter vor, von $\overline{\mathrm{P}} \infty$ (alle 4 Flächen) und $\infty \overline{\mathrm{P}} \infty$ begrenzt, und $\overline{\mathrm{P}} \infty: \overline{\mathrm{P}} \infty$ 
362 Jorgensen: Ueber den sogenannten Herapathit

ist $=103^{\circ}$ gemessen; bei dem Sulfat kommen $\overline{\mathrm{P}} \infty$ (mit 2 parallelen Flächen) und $\infty \overline{\mathrm{P}} \infty$ vor, und $\overline{\mathrm{P}} \infty: \overline{\mathrm{P}} \infty$ ist $=111,33^{\circ}$ gefunden. Mit $\overline{\mathrm{P}} \infty$ bei dem Seleniat als Grundform wird nun die entsprechende Form beim Sulfate zwar $=1 / 2 \bar{P} \infty\left(\right.$ Rechn.$\left.=111^{\circ} 40^{\prime}\right) . \quad$ Aber hier sollte dann das eigenthümliche Verhalten stattfinden, dass das Seleniat die optische Wirkung: || undurchsichtig, + hell olivengelb, das Sulfat aber die entgegengesetzte zeigen sollte, was mir im höchsten Grade unwahrscheinlich vorkommt.

Dass die Verbindung unverändertes Chinidin enthält, wurde auf dieselbe Weise wie bei dem ersten Chinidinperjodidsulfat dargethan. In dem schwer löslichen jodwasserstoffsauren Salze wurden 27,97 Proc. Jod gefunden (Rechn. 28,09).

Auch lässt sich diese Verbindung leicht in die vorhergehende umwandeln. Dadurch wird zugleich dargethan, dass diese $z$ wei Verbindungen nicht, wie Herapath annahm, zwei verschiedene Chinidinmodificationen enthalten. Löst man nämlich das zweite Salz in heissem Weingeist unter Zusatz von etwas Jodwasserstoffsäure, so scheiden sich beim Verdünnen mit etwas Wasser, Erkalten und Stehen rothbraune. ziemlich kurze Prismen des ersten Chinidinperjodidsulfats mit allen seinen physikalischen Eigenschaften ab (gef. 49,82 Proc. Jod, 33,2 freieres Jod; Rechn. $50,46$ und 33,65$)$.

Vom 2ten Chinidinperjodidsulfat wird kalter Schwefelkohlenstoff kaum, siedender schwach, Chloroform und Aether nicht gefärbt. Es löst sich ziemlich leicht in heissem Weingeist. Schon beim Schütteln mit kaltem Weingeist und Quecksilderjodiddoppelsalz, in heissem Weingeist löslich, beim Erkalten sich in amorphen Kügelchen abscheidend.

Die ursprüngliche Verbindung lässt sich nicht umkrystallisiren, wenigstens nicht aus siedendem Weingeist. Bei langsamem Erkalten scheidet die Lösung theils ein rothbraunes Pulver ab, mit schwarzen halbfin̈ssigen Massen gemischt, theils Gruppen diamantglänzender, branner Prismen 
mit blauem Reflex, dem 1 ten Chinidinperjodidsulfat sehr ähnlich und auch fast dieselbe Menge freieres Jod haltend (gef. 34,3; Rechn. 33,65 Proc.). Jedoch bestehen sie aus einer ganz verschiedenen Verbindung. Schon beim Waschen mit kaltem, verdünntem Weingeist verlieren sie den Glanz, und sie wirken auf polarisirtes licht sehr kräftig ein: \| der Längsaxe schwarzbraun bis undurchsichtig, + gelbbraun bis braun. Diese Verbindung habe ich leider nicht, näher untersucheu können. Erstens wird sie in verhältnissmässig geringer Menge gebildet; dann erscheint es kaum möglich, dieselbe vollständig von den gleichzeitig gebildeten Substanzen zu trennen.

\section{E. Aethylehinidinverbindung.}

Versetzt man eine weingeistige Lösung von Chinidin mit Jodäthyl und reichlichem Aether, so wird die Flasche beim Stehen mit farblosen, mehrere $\mathrm{Cm}$. langen, seideglänzenden Nadeln von Jodäthylchinidin angefüllt. Zu den von Stenhouse ${ }^{1}$ ) gegebenen, ziemlich spärlichen Mittheilungen von den Aethylchinidinverbindungen kann ich Folgendes hinzufügen.

Durch Digeriren des Jodids mit Chlorsilber und Wasser erluält man eine Lösung des neutralen Chlorids. Dieselbe verhält sich gegen Reagentien in folgender Weise : Natron, Ammoniak und kohlensaure Alkalien sind ohne fällende Wirkung. - Platinchloridlösung fällt blass orangefarbenes, fast unlösliches Platindoppelsalz, aus äusserst kleinen Krystallen bestehend. Aus stark verdünnten, stark salzsauren und siedenden Lösungen erhält man deutlichere Krystalle von etwas dunklerer Farbe. Bei $120^{\circ}$ getrocknet hält das Doppelsalz 25, 70 Proo. Platin (Rechn. für $\mathrm{C}_{20} \mathrm{H}_{24} \mathrm{~N}_{2} \mathrm{O}_{2}$, $\mathrm{C}_{2} \mathrm{H}_{5}$. $\mathrm{HPt} \mathrm{Cl}_{6}=25,81$ ). - Goldchlorid erzeugt einen gelben, körnigen Niederschlag, bei Erwärmen sich zum Theil lösend, zum Theil aber zu einem braungelben Oel schmelzend. Das friscb gefällte Salz löst sich in Weingeist. Quecksilberchlorid giebt einen weissen, fast unlöslichen

1) Lond. Roy. Soc. Proceed. 12, 491. 
364 Jorgensen: Ueber den sogenannten Herapathit

Niederschlag, der bei Erwärmen unter der Flüssigkeit zu farblosem Oel schmilzt. Der kalt gefällte Niederschlag löst sich leicht in erwärmter verdünnter Salzsäure. Beim Erkalten schiessen dann farblose Nadeln an. - Kaliumquecksilberjodid liefert einen weissen voluminösen Niederschlag; aus heisser weingeistiger Lösung scheiden sich blassgelbe Nadeln aus. - Wasserstoffwismuthjodid fällt in kalten wässrigen oder in heissen weingeistigen Lósungen scharlachroth, amorph. - Jodkalium fällt das in kaltem Wasser sehr schwer lösliche, in heissem lösliche Jodid. Beim Erkalten krystallisirt es in prachtvollen, seidenglänzenden, doppelbrechenden $\mathrm{Nadeln}$ von mehreren $\mathrm{Cm}$. Länge. - Kaliumperjodid erzeugt in heissen, weingeistigen Lösungen lange braune Nadeln des Trijodids. - Sulfocyankalium giebt einen weissen, voluminösen, krystallinischen Niederschlag, in siedendem Wasser löslich, daraus beim Erkalten in feinen, diamantglänzenden, doppelbrechenden Nadeln krystallisirend. - Kaliumchromat fällt nicht, Kaliumdichromat giebt einen gelben voluminösen, schnell sich zu Klumpen zusammenballenden Niederschlag. - Saures Silbersulfat liefert ein stark fluorescirendes Filtrat.

$$
\begin{aligned}
& \text { Aethylchinidıneerjodidsulfat. } \\
& 2 \mathrm{C}_{20} \mathrm{H}_{24} \mathrm{~N}_{2} \mathrm{O}_{2}\left(\mathrm{C}_{2} \mathrm{H}_{3}\right) \mathrm{J}, \mathrm{SH}_{2} \mathrm{O}_{4}, \mathrm{~J}_{4} \text {. }
\end{aligned}
$$

Löst man 2 Mol. Jodäthylchinidin in $1 \mathrm{Mol}$. normaler Schwefelsänre $(=20 \mathrm{Ccm}$.) in $50 \mathrm{Ccm}$. heissem Weingeist von $93^{\circ} \mathrm{T}$. und versetzt die Lösung mit $4 \mathrm{At}$. Jod, in warmem Weingeist gelöst, so krystallisirt bei langsamem Erkalten und Stehen das Acidperjodid in ziemlich grossen Krystallen, und zwar so vollständig, dass man ohne Schwierigkeit bis 97 Proc. der theoretischen Menge gewinnt. Die Krystalle sind dunkelbraune, diamant-, fast metallglänzende Prismen, jedoch sind sie häufig gestreift und canellirt, und etwa vorhandene Pyramiden und Domen immer undeutlich ausgebildet. Im Aeussern ist diese Verbindung kaum vom 1ten Chinidinperjodidsulfat zu unterscheiden. Sie absorbirt schwach das polarisirte Licht: I dunkler, 
+ heller braun. Die lufttrockne Verbindung verliert neben Vitriolöl kein Wasser.

Die Analyse ergab:

$\begin{array}{lrcc} & \text { Rechn. } & \text { a. } & \text { b. } \\ \mathrm{SO}_{3} & 5,11 & 5,20 & - \\ \mathbf{J} & 48,66 & 48,60 & \mathbf{4 8 , 5 7} \\ \text { Freieres Jod } & \mathbf{3 2 , 4 4} & - & \mathbf{3 2 , 4 0}\end{array}$

Die rationelle Formel entspricht ganz der des 1 ten Chinidinperjodidsulfats. Die Verbindung löst sich sehr schwer in kaltem, schwer selbst in siedendem Weingeist. Sie färbt nicht kaltes oder siedendes Chloroform, Aother oder Schwefelkohlensfoff. Das Quecksilberjodiddoppelsalz (bei dessen Bildung kein Quecksilberjodür entsteht) lüst sich schwer in kaltem, weit leichter in siedendem Weingeist und krystallisirt beim Erkalten, in hellgelben, klaren, anscheinend rhombischen Prismen, ofımals zu Bündeln vereinigt. Mit Silbernitrat bildet das Perjodidsulfat sowohl Silberjodat wie Jodsilber.

F. Cinchoninverbindungen.

1 tes Cinchoninperjodidsulfat. $8 \mathrm{C}_{20} \mathrm{H}_{24} \mathrm{~N}_{2} \mathrm{O}, 6 \mathrm{SH}_{2} \mathrm{O}_{4}, 6 \mathrm{HJ}, \mathrm{J}_{10}, 12 \mathrm{H}_{2} \mathrm{O}$.

Diese Verbindung wird leicht erhalten beim Auflösen von $2 \mathrm{Mol}$. Cinchonin, $1 \mathrm{Mol}$. Schwefelsäure und $2 \mathrm{Mol}$. Jodwasserstoffsäure in heissem Weingeist und Zusatz von 2 At. Jod in heisser, weingeistiger Lösung. Hat man nicht mehr als $100 \mathrm{Ccm}$. Weingeist auf jedes Mol. Cinchonin $(=3,08 \mathrm{Grm}$.) angewandt, so erstarrt beim Erkalten die ganze Flüssigkeit zu einem Magma von braunen, wolligen, höchst undeutlichen Warzen, die selbst unter dem Mikroskop kaum Spuren krystallinischer Structur darbieten. Beim Waschen mit schwachem Weingeist $\left(50^{\circ} \mathrm{T}\right.$.) wird die Verbindung schon theilweise zersetzt unter Bildung des folgenden Acidperjodids, und die Zersetzung schreitet in diesem Falle beim Trocknen neben Vitriolöl noch weiter fort. Daher ist die Verbindung mit eiskaltem, starkem Weingeist zu waschen, sogleich umzukrystallisiren, 
366 Jorgensen: Ueber den sogenannten Herapathit

zu pressen und neben Vitriolöl zu trocknen. Wegen der ungemein voluminösen Form dieser Verbindung wäscht man zweckmässig durch Decantation. Um vollständiges Trocknen zu erreichen, ist es nothwendig, nach Trocknen bis zu constantem Gewicht die Verbindung zu pulverisiren und dann wieder zu trocknen.

Das neben Vitriolöl völlig getrocknete Salz verliert die Hälfte seines Wassers bei etwa $100^{\circ}$ in trocknem Luftstrom (es beginnt schon bei etwa $70^{\circ}$ Wasser abzugeben); bei $120^{\circ}$ fängt es wieder an Wasser zu verlieren; dieser Wasserverlust ist bei $130^{\circ}$ vollständig vorüber; bei $140^{\circ}$ bis $145^{\circ}$ schmilzt die Verbindung und schon bei 160 bis $170^{\circ}$ wird sie vollständig unter Entwicklung von Chinolin zersetzt.

Die Analyse der neben Vitriolöl getrockneten Verbindung ergab:

\begin{tabular}{|c|c|c|c|c|c|c|}
\hline & & & & efund & & \\
\hline & Rechn. & a.1) & b. & c. & d. & d. \\
\hline $\mathrm{C}$ & 36,21 & - & - & - & 36,04 & - \\
\hline $\mathbf{H}$ & 4,41 & - & - & - & 4,45 & - \\
\hline $\mathrm{SO}_{3}$ & 9,05 & 8,92 & 9,07 & - & 9,18 & - \\
\hline $\mathbf{J}$ & 38,27 & 38,96 & 38.09 & 38,08 & - & - \\
\hline Freieres Jod & 23,94 & 24,80 & - & 24,10 & 24,10 & - \\
\hline Wasser bei $100^{\circ}$ & 2,03 & -- & 1,86 & - & 1,89 & 1,83 \\
\hline$" \quad, 130^{\circ}$ & D 2,03 & - & $\ldots$ & - & 2,19 & - \\
\hline
\end{tabular}

Eine rationelle Formel, welche zugleich die Jeichte Umbildung der Verbindung in die folgende berücksichtigt, wird sein:

$$
\begin{aligned}
& \mathrm{HO} . \mathrm{SO}_{2} \cdot \mathrm{O} . \mathrm{HCiH} \cdot \mathrm{O} . \mathrm{SO}_{2}, \mathrm{O} . \mathrm{H} \mathrm{CiHJ} \\
& J_{2} \text { J } J_{2} . J . H C i H . O . \mathrm{SO}_{2} \text {.O.HCiH.J. } J_{2} \\
& \mathrm{~J}_{2} \text {.J.H Ci H.O. } \mathrm{SO}_{2} \text {. O.H Ci H.J. }
\end{aligned}
$$

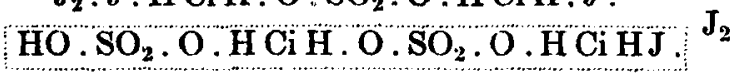

Beim Behandeln mit schwachem Weingeist oder bei langem Stehen unter der Mutterlauge wird die Verbindung nämlich so verändert, dass die eingeklammerten Atom-

1) Dies Präparat hielt Spuren der folgenden Verbindung. 
complexe (saure Cinchoninsalze) weggespalten werden unter Hinterlassung der folgenden Verbindung. Letztere tritt übrigens hier, besonders bei längerem Stehen, gebildet in schönen, fast schwarzen, glasglänzenden Krystallen auf (gef. 39,6 Proc. freieres Jod; Rechn. 39,56), die beim langen Stehen an Glanz verlieren.

lstes Cinchoninperjodidsulfat ist ziemlich schwer in kaltem, äusserst leicht in heissem Weingeist löslich, daraus unverändert krystallisirbar. Es färbt weder Aether, Chloroform, noch Schwefelkohleustoff. Die weingeistige Lösung liefert, mit Quecksilber geschüttelt, kein Quecksilberjodür, sondern ein weissgelbes, auch in siedendem Weingeist ziemlich schwer lösliches Quecksilberjodiddoppelsalz, das beim Erkalten in derselben Form, wie die Quecksilberjodiddoppelsalze der anderen Cinchoninperjodidsulfate krystallisirt $\left(\overline{\mathrm{P}} \infty: \infty \overline{\mathrm{P}} \infty\right.$ gef. $=136^{\circ}$ bis $\left.136,5^{\circ}\right)$. Auch das Thalliumjodiddoppelsalz ist dem der folgenden Verbindung ganz ähnlich. Wahrscheinlich ist daher die Verbindung in heisser weingeistiger Lösung zersetzt, bindet sich aber wieder bein Erkalten.

$$
\begin{aligned}
& \text { 2tes Cinchoninperjodidsulfat. } \\
& 4 \mathrm{C}_{20} \mathrm{H}_{24} \mathrm{~N}_{2} \mathrm{O}, 2 \mathrm{SH}_{2} \mathrm{O}_{4}, 4 \mathrm{HJ}, \mathrm{J}_{10} \text {. }
\end{aligned}
$$

Man löst Cinchonin in siedendem Weingeist unter Zusatz der berechneten Mengen Schwefelsäure und Jodwasserstoffsäure, aber auf jedes Mol. Cinchonin höchstens 2 At. Jod in warmer woingeistiger Lösung. Bei langsamem Erkalten krystallisiren dann, nach der Concentration der Aufösung, dünne, branne, diamantglänzende Blätter oder dickere, fast schwarze Prismen. Im Mikroskop erscheinen erstere rechtwinklig. Dünnure Blätter zeigen das optische Verhalten: \| dunkelbraun bis braun, + hellbraun bis braungelb, je nach der Dicke. Dickere Exemplare sind fast ganz undurchsichtig.

Durch das freundliche Wohlwollen des Hrn. Dr. Topsó kann ich folgende krystallographische Mittheilungen von dem aus der vorhergehenden Verbindung duroh längeres 
368 Jorgensen: Ueber den sogenannten Herapathit

Stehen unter der Mutterlange gebildeten Salze hinzufügen:

„Monoklinisch. Die Krystalle sind kleine, 4 seitige Tafeln, das Flächenpaar (100) von Randkantenflächen, den Formen (110) . (001) . (101) gehörend, begrenzt. Die Flächen spiegeln sebr schlecht, die Messungen sind daher ganz approximativ.

$$
\left\{\begin{array} { l } 
{ 1 0 0 : 1 1 0 \text { c. } 5 5 ^ { 0 } } \\
{ 1 1 0 : \overline { 1 } 1 0 \text { c. } 7 0 ^ { 0 } }
\end{array} \left\{\begin{array}{ll}
100: 001 & \text { c. } 88^{3} / 4^{0} \\
100: \overline{1} 01 & 42^{0} \\
001: \overline{1} 01 & \text { c. } 54^{1} / 4^{0}
\end{array}\right.\right.
$$

„Guter Durchgang parallel dem Flächenpaar (100)."

Versucht man die Verbindung umzukrystallisiren, so erhält man ein Gemenge der vorhergehenden und der folgenden Verbindung, möglicher Weise bildet sich zugleich Cinchonintrijodid. ${ }^{1}$ )

Die Analyse der neben Vitriolöl getrockneten Verbindung ergab:

\begin{tabular}{lcrcc} 
& Rechn. & \multicolumn{3}{c}{ Gefiunden } \\
$\mathrm{SO}_{3}$ & 4,99 & 5,12 & - & - \\
$\mathrm{J}$ & 55,39 & 55,09 & 55,24 & 55,23 \\
Freieres Jod & 39,56 & - & 39,70 & -
\end{tabular}

Verbrauchtes Thalliumjodür auf $1 \mathrm{Mol}$. der Verbindung gef. 5,04 Mol. (Rechn. 5).

Daraus folgt die wahrscheinliche rationelle Formel:

$$
\begin{aligned}
& \mathrm{J}_{2} . \mathrm{J} . \mathrm{H} \mathrm{CiH} . \mathrm{O} . \mathrm{SO}_{2} . \mathrm{O} . \mathrm{H} \mathrm{CiH} . \mathrm{J} . \mathrm{J}_{2} \cdot \mathrm{J}_{2} \text {. } \\
& \mathrm{J}_{2} \text {.J.H Ci H.O. } \mathrm{SO}_{2} . \mathrm{O} . \mathrm{HCiH} . \mathrm{J} . \mathrm{J}_{2} \cdot
\end{aligned}
$$

Die Verbindung färbt nicht kalten, kaum siedenden Aether; kalter Schwefelkohlenstoff wird kaum, siedender schwach gefärbt; Chloroform färbt sich weder in der Kälte, noch beim Sieden. Mit Quecksilber bildet die weingeistige Lösung Jodür und ein Jodiddoppelsalz, das sich beim $\mathbf{E r}$ kalten der filtrirten Flüssigkeit als ein kaum krystallinischer, weissgelber Niederschlag, welcher sehr fest an den Gefässwänden haftet, absetzt. Wird dasselbe in siedendem Weingeist, worin es ziemlich schwer löslich, gelöst, so

1) Dies Journ. [2] 8, 148. 
scheiden sich bei langsamem Erkalten Rosetten diamantglänzender, fast farbloser, centimeterlanger, rhombischer Blätter ab $\left(\overline{\mathrm{P}} \infty: \infty \overline{\mathrm{P}}^{\circ} \infty\right.$ gef. $\left.=136^{\circ}\right)$. Das Thalliumjodiddoppelsalz bildet grosse, scharlachrothe, unregelmässig ausgebildete Prismen und federförmige Aggregate; bei schnellem Erkalten werden orangegelbe, eigenthümlich entwickelte, ästige Aggregate von Prismen und Blätter erhalten, welche jedoch beim Stehen unter der Flüssigkeit in das eben exwähnte scharlachrothe Salz übergehen. Das gepulverte Acidperjodid liefert, mit wässrigem Silbernitrat erwärmt, ein Gemenge von Jodsilber und Silberjodat.

3 teo Cinehoninperjodidoulfat.

$$
2 \mathrm{C}_{20} \mathrm{H}_{24} \mathrm{~N}_{2} \mathrm{O}, \mathrm{8H}_{2} \mathrm{O}_{4}, 2 \mathrm{HJ}, \mathrm{J}_{6} \text {. }
$$

Diese, wahracheinlich mit dem von Herapath erhaltenen Cinchoninsalz identische Verbindung erhielt ich nach folgenden Weisen: 1) Man löst Cinchonin in den berechneten Mengen normaler Schwefelsäure und schwachem Jodwasserstoff, versetzt mit Weingeist, erwärmt, jedoch nicht stark, und fügt die berechnete Menge heisser Jodtinctur binzu. Bei langsamem Erkalten uad Stehen scheiden sich dann schwarze Prismen ab, zu breiten Blättera mit bläulichem Reflex parallel zusarnmengewachsen. So wird fast die theoretische Menge der Verbindung erhalten; aus 3,08 Grm. Cinchonin erhielt ich 8,21 Grm. (Rechn. 8,66) der reinen Verbindung. - 2) Man löst das vorhergehende Salz in heissem Weingeist und versetzt mit Wasser in kleinen Antheilen und unter Umrühren, oder 3) man löst die theoretischen Mengen der Bestandtheile in heissem Weingeist und fällt ebenso mit Wasser. Auch so habe ich fast die berechnete Menge erhalten $(95,8$ Proc.).

Die Analysen des neben Vitriolöl getrockneten Salzes haben ergeben: 
370 Jorgensen: Ueber den sogenannten Herapathit

$\begin{array}{lrcccc} & \text { Rechn. } & 1 . & 2 . & 3 . & \begin{array}{c}\text { Herapath } \\ \text { fand im Mittel. }\end{array} \\ \mathbf{C} & \mathbf{2 7 , 7 2} & 27,71 & - & \mathbf{2 7 , 4 8} & \mathbf{2 7 , 7 0} \\ \mathrm{H} & \mathbf{3 , 0 0} & \mathbf{3 , 0 8} & - & 3,22 & \mathbf{3 , 4 9} \\ \mathrm{N} & \mathbf{3 , 2 3} & - & - & - & \mathbf{3 , 3 1} \\ \mathrm{SO}_{3} & \mathbf{4 , 6 2} & \mathbf{4 , 7 2} & - & \mathbf{4 , 7 2} & \mathbf{5 , 2 3} \\ \mathrm{J} & \mathbf{5 8 , 6 6} & \mathbf{5 8 , 6 5} & \mathbf{5 8 , 3 0} & \mathbf{5 8 , 4 0} & \mathbf{5 0 , 4 1} \\ \text { Freieres Jod } & \mathbf{4 3 , 4 9} & - & \mathbf{4 3 , 3 0} & \mathbf{4 3 , 9 0} & -\end{array}$

Verbrauchtes Thalliumjodür auf 1 Mol. gef. 3,04 (Rechn. 3).

Die wahrscheinliche rationelle Formel ist:

$$
\mathrm{J}_{2} \text {.J.HCiH.O. } \mathrm{SO}_{2} \text {. O. H Ci H.J.J4. }
$$

Herapath's Analysen zeigen die gewöhnlichen Fehler für Schwefelsäure und Jod, während seine Zahlen für Kohlenstoff, Wasserstoff und Stickstoff meiner Formel vollständig entsprechen.

Die Verbindung färbt kaum kalten Aether, siedender wird schwach, kalter Schwefelkohlenstoff deutlich, siedender etwas stärker, kaltes Chloroform äusserst schwach, siedendes etwa wie kalter Schwefelkohlenstoff yefärbt. In heissem Weingeist löst sich das Salz ziemlich leicht. Die weingeistige Lösung liefert, mit Quecksilber geschüttelt, viel Jodür und ein Jodiddoppelsalz von ganz denselben Eigenschaften wie das aus der vorhergehenden Verbindung entstandene. Auch die Thalliumsalze scheinen identisch. Ebenso ist das Verhalten gegen Silbernitrat das nämliche.

Die Verbindung enthält, der Ansicht Herapath's entgegengesetzt, unverändertes Cinchonin. Die Lösung des gepulverten Acidperjodids in verdünnter wässriger schwefliger Säure giebt mit Ammoniak einen völlig farblosen krystallinischen Niederschlag von Cinchonin, das nach Wiederauflösen in verdünnter Salzsäure und Fällen mit Ammoniak, um Spuren ron Jodwasserstoff zu entfernen, ein Platinchloriddoppelsalz von 27,41 Proc. Platingehalt (Rechn. 27,35), so wie das für Cinchonin charakteristische Trijodid in aller Schönheit dieser Verbindung lieferte. 
G. Cinchonidinverbindungen.

Unter Cinchonidin wird hier das Cinchonidin von Pasteur, das Chinidin von Hesse verstanden. Das zu nachstehenden Untersuchungen verwendete Alkaloid warde grossen Theils aus dem charakteristischen schwer löslichen Tartrat, theilweise auch aus dem messinggelben Perjodidsulfat dargestellt.

1 tes Cinchonidinperjodidsulfat. $12 \mathrm{C}_{20} \mathrm{H}_{24} \mathrm{~N}_{2} \mathrm{O}, 9 \mathrm{SH}_{2} \mathrm{O}_{4}, 8 \mathrm{HJ}, \mathrm{J}_{24}, 8 \mathrm{H}_{2} \mathrm{O}$.

Diese Verbindung (Herapath's messinggelbes Salz) wird durch langsames Erkalten einer Lösung der theoretischen Mengen der Bestandtheile in heissem Weingeist leicht erhalten. Sie krystallisirt dann in ungemein schönen, mehrere Mm. langen, rhombischen, goldgrünen Blättern mit einem, im Gegensatz zu dem grasgrünen des Herapathits, hervortretenden messinggelben Glanz. Auch bei Anwendung von nur $6 \mathrm{Mol}$. Jodwasserstoff auf $12 \mathrm{Mol}$. Cinchonidin wird die Verbindung erhalten, selten aber in so schön ausgebildeten, auch nicht in so grossen und glinzenden Formen. Ueberhaupt wird diese Verbindung am sichersten und schönsten bei Gegeuwart etwas überschüssiger Schwefelsäure gebildet. Sie scheint von den 4 ron mir untersuchten Cinchonidinperjodidsulfaten die zu sein, welche besonders bei höherer Temperatur gebildet wird. Denn selbst bei so abweichenden Verhältnissen, wie $12 \mathrm{Ci}: 6 \mathrm{SH}_{2} \mathrm{O}_{4}: 9 \mathrm{HJ}: 18 \mathrm{~J}$, fängt sie in der Nähe des Siedepunktes der Flüssigkeit an, sich zu bilden. Jedoch wird sie dann bei langamem Erkalten ziemlich scinell in eine Verbindung verwandelt, die grosse äussere Aehnlichkeit mit den später zu beschreibenden olivengrïnen Nadeln zeigt. Ob wirklich letzteres Salz hier entsteht, kann nicht mit Sicherheit entschieden werden, weil auch die olivengrünen Nadeln unter diesen Verhältnissen noch vor dern vollständigen Erkalten der Flüssigkeit in eine dritte Verbindung (gelbe glänzende Nadeln) übergehen; jedoch ist letztere Veränderung erst nach einigen Tagen vollständig. 
372 Jorgensen: Ueber den sogenanuten Herapathit

Worden die drei unten zu beschreihenden Cinchoninperjodidsulfste in siedendem Weingeist gelöst, so krystallisirt ebenfalls aus der fast siedenden Flüssigkeit, besonders bei starkem Umrühren und Reiben der Gefässwände, das messinggelbe Salz, welches jedoch unter diesen Umstinden ziemlich sohnell wieder auf versehiedene Weise umgebildet wird, worüber unten Näheres.

Die völlig metallglänzenden Blätter des ersten Cinchonidinperjodidsulfats sehen dem krystallisirten Messing sehr ahnlich. Bei langsamem Erkalten ausgeschieden, zeigen sie nur P $\infty$ oder $\overline{\mathrm{P}} \infty .0 \mathrm{P}$. Letztere Form tritt bisweilen so stark hervor, dass die Krystalle die Form von flachen Nadeln erhalten. Bei schnellem Erkalten oder aus einer Flüssigkeit, die ein vom Sala selbst viel abweichendes Verhältniss der Bestandtheile has, erhält man sie gewöhnlich äusserst dünn, zerrissen oder höchst unregelmässig ausgebildet und zusammengewachsen. $\overline{\mathrm{P}} \infty \mathrm{O}: 0 \mathrm{P}$ wurde in 3 Messungen an einem Krystall $=111,7^{\circ}$, in 3 an einem anderen $=112^{\circ}, \overline{\mathrm{P}} \infty: \overline{\mathrm{P}} \infty$ im Mittel von 23 Messungen an 6 Krystallen von 4 Präparaten $=43,9^{\circ}$ gefunden. Die Blätter wirken äusserst kräftig polarisirend: der Axe undurehsichtig, in sehr dünnen Blättern dunkelblau, + hell olivengelb bis olivenbräunlich, in sehr dünnen Blättern fast farblos.

Bei wiederholtem Umkrystallisiren vorliert das Salz ein wenig Jod und Schwefelsäure, wird dabei weniger metallglänzend, mehr gräulich nnd krystallisirt schliesslich in undeutlichen Warzen. Dagegen lässt es sich unverandert mit sohwachem Weingeist waseben, ja aus der weingeistigen Lösung lässt es sich unveründert durch Wasser fällen und unterscheidet sich Jadurch günzlich vom entsprechenden Seleniat (s. u.).

Die Analysen des neben Vitriolöl getrockneten Balzes ergaben : 
und ahnliche Acidperjodide.

\begin{tabular}{|c|c|c|c|c|c|c|c|c|c|c|}
\hline & Rechn. & a. & a. & b. & c. & $a$ & d. & $d^{\prime}$ & $d^{2}$ & e. \\
\hline C & 32,75 & - & - & 31,80 & 82,00 & - & - & - & - & - \\
\hline $\mathbf{H}$ & 3,75 & 3,91 & - & 3,85 & 4,01 & - & - & - & - & - \\
\hline $\mathrm{SO}_{3}$ & 8,19 & 8,32 & $\rightarrow$ & - & 8,11 & - & 8,21 & - & - & 8,18 \\
\hline $\mathbf{J}$ & 46,21 & 46,39 & 46,23 & 46,36 & 46,46 & - & 46,34 & - & 45,45 & 46,84 \\
\hline Freieres & J 34,66 & 34,50 & - & - & 34,70 & 34,60 & - & 84,30 & 38,80 & - \\
\hline $\mathrm{H}_{2}$ O.b.1c & $10^{0} 1,64$ & - & - & - & 1,77 & - & 1,67 & - & - & - \\
\hline
\end{tabular}

Verbrauchtes Thalliumjodür auf $1 \mathrm{Mol}$ gaf. 12,19 Mol. (Rechn. 12).

Das unter $d^{\prime}$ war 2 Mal, das unter $d^{2} 3$ Mal umkrystallisirt worden.

Die ungemein complicirte Formel, zu welcher die gefundenen Zahlen führten, oo wie der Umstand, dass $\mathrm{He}$ rapath, der die Verbindung entdeckt hat, nicht nur wie gewöhnlich allzu wenig Jod (im Mittel 39,23 Proc.) und za viel Schwefelsäure (im Mittel 8,63 Proc.), sondern auch sehr abweichende Zahlen für Kohlenstoff (im Mittel 35,49) und Wasserstoff (im Mittel 4,31) gefunden hat, waren die Ursache, warum diese Verbindung so häufig dargestellt und analysirt wurde. In Bezug anf die Schwefelsärre und das Jod weise ich auf meine allgemeinen Bemerkungen zu Herapath's Analysen hin. Wenn aber Herapath's Kohlen- und Wasserstoffbestimmungen hier zu hoch ausgefallen sind, so hat dies einen besonderen Grund. Das von ihm analysirte Präparat wurde nämlich ,at $212^{\circ} \mathrm{F}$. during 4 houres, on till the weight remained constant" getrocknet. Aber bei 4 stündigem Erwärmen auf $100^{\circ}$ verliert die Verbindung, je nach der in Arbeit genommenen Monge, 5 bis 7 Proc. im Gewicht, indem sie gleichzeitig Wasser und Jod verliert; auch bört sie nicht nach 4 Stunden auf an Gewicht zu verlieren, für jede weitere Stunde erfihrt sie einen Gewiohtsverlust von ein paar Milligramm. Ich habe so das Salz über 11 Proc. im Gewicht verlieren sehen; dann brach ich den Versuch ab, der mir zu keinem Resultat führen zu können schien. Herapath hat daher eine theilweise zersetzte Verbindung analysirt, wurde aber darauf nicht aufmerksam, weil die Substanz auch nach dem Trocknen bei $100^{\circ}$ nicht viel ihr Ansehen verändert. 


\section{Jorgensen: Ueber den sogenannten Herapathit}

- Dass die von mir analysirten Präparate kein freies Jod enthielten, zeigte sich dadurch, dass sie, auch in fein gepulvertem Zustande mit kaltem Schwefelkohlenstoff geschüttelt, denselben nicht oder doch nur ganz verschwindend färbten; selbst kochender Schwefelkohlenstoff färbte sich äusserst schwach, Aether oder Chloroform gar nicht, selbst beim Kochen.

Mit Quecksilber geschüttelt liefert die heisse weingeistige Lösung Quecksilberjodür, das Filtrat setzt beim Erkalten weisse Warzen eines Quecksilberjodiddoppelsalzes ab, das unter dem Mikroskop als äusserst kleine Nadeln erscheint. Das Perjodidsulfat löst sich schwer in kaltem, ziemlich leicht in kochendem Weingeist. Beim Zuratz von Jodwasserstoffsäure löst es sich sehr leicht in warmem Weingeist, beim Erkalten soheidet sich gewöhnlich nichts ab, erst beim Stehen bilden sich die langen olivengrünen Nadeln des 3 ten Cinchonidinperjodidsultats, die allmählich die ganze Flüssigkeit erfüllen und nach und nach in 2 tes Cinchonidinperjodidsulfat übergehen.

$$
\begin{gathered}
2 \text { tes Cinchonidinperjodidsulfat. } \\
4 \mathrm{C}_{20} \mathrm{H}_{24} \mathrm{~N}_{2} \mathrm{O}, 2 \mathrm{SH}_{2} \mathrm{O}_{4}, 3 \mathrm{HJ}, \mathrm{J}_{6}, 4 \mathrm{H}_{2} \mathrm{O} \text {. }
\end{gathered}
$$

Diese Verbindung scheidet sich fast immer aus der Mutterlauge der vorhergehenden und der nachfolgenden Verbindung als lange, dünne, seidenglänzende, rothgelbe, oft fast goldgelbe Nadeln. Unter dem Mikroskop zeigen sie sich als parallel der Axe gestreifte Prismen, deren Enden immer undeutlich modificirt sind, und die das Licht nur scbwach polarisiren: "I der Axe bellgelb, + braungelb. Obwohl aber diese Verbindung oft in der Mutterlauge der vorhergehenden ersoheint (bisweilen geht letztere unter der Mntterlauge allmählioh vollatändig in diese über) und obwohl diese Mutterlauge immer sauer ist, so beruht die Bildung der gelben Nadeln keineswegs, wie Herapath annimmt, suf die Gegenwart überschüssiger Schwefelsäure. Man erhält im Gegentheil immer am leichtesten und sichersten die gelben Nadeln aus einer Flüssigkeit, die weniger 
Schwefelsäure enthält, als der Zusammensetzung des messinggelben Salzes entspricht; auch enthalten die gelben Nadeln weit weniger Schwefelsäure als das messinggelbe Salz. Dagegen ist es wichtig, dass die Mutterlauge sauer ist, am besten jodvasserstoffsauer, weil 1 tes Cinchonidinperjodidsulfat weit leichter in jodwasserstoffsanrem Weingeist als in reinem löslich ist. Dies beruht nämlich, wie oben angedeutet, darauf, dass es hierdurch in olivengrünes Perjodidsulfat übergeht, und letzteres verwandelt sich weit Jeichter als das messinggelbe Salz in die gelben Nadeln. Dies Verhalten musste aber Herapath unbekannt bleiben, weil er das olivengriue Salz nicht gekannt hat, und zwar leshalb, weil er nie Jodwasserstoff, nur Jodtinctur allein zu seinen Präparaten anwendete. Zwar erwähnt auch er ein olivengrünes $\mathrm{Salz}$, dies ist aber etwas ganz anderes als mein 3 tes Cinchonidinperjodidsulfat, was aus dem Folgenden erhellen wird.

Die gelben Nadeln werden zwar aus den berechneten Mengen Cinchonidin, Schwefelsäure, Jodwasserctoff und Jod in heisser weingeistiger Lösung erhalten, am besten, indem man auf $1 \mathrm{Mol} .=3,08 \mathrm{Grm}$. Cinchonidin etwa $150 \mathrm{Ccm}$. Weingeist von $90^{\circ} \mathrm{T}$. anwendet (incl. den zur Auflösung des Jods gebrauchten). Indessen bilden sich hier zuerst, während die Flüssigkeit noch heiss ist, die messinggelben Blätter und bei weiterem Erkalten die olivengrünen Nadeln. Bei vollständigem Erkalten und besonders bei einigen Tagen Stehen werden nun zwar die letzteren in die gelben Nadeln verwandelt, und diese Umbildung ist eine vollständige, dagegen kaun es sehr lange (mebrere Monate) dauern, bevor die letzten Spuren ler messinggelben Verbindung verschwinden. Filtriri man dagegen die Flüssigkeit, sobald die olivengrünen Nadein sich zu bilden anfangen, so giebt das Filtrat entweder nur die gelben Nadeln, oder wenn sich anch anfangs olivengrünes Salz abschridet, so wird letzteres nach wenjgei als 24 Stunden vollständig zu den gelben Nadeln verwandelt. Auoh wenn man Cinchonidin, Schwefelsüure und Jod in hereohneten Mengen, aber statt 3 Mol. Jodwasserstoff 
4 Mol. anwendet, erhält man, besonders wenn man die Temperatur nicht $60^{\circ}$ übersteigen lässt, gar kein messinggelbes Salz gebildet, und zwar aus oben angeführten Gründen, sondern ein Gemenge des gelben und olivengrünen Salzes, das bei einigen Tagen Stehen unter der Mutterlauge vollständig zu gelben Nadeln wird, welch letztere bei der etwas verlangsamten Bildung sehr schön, mehrere $\mathrm{Cm}$. lang und gewöhnlich etwas dicker und daher dunkler wie gewöbnlich sind.

Aus Gemengen, welche die gelben Nadeln mit mehr oder weniger der übrigen Cinchoninperjodidsulfate enthalten, können erstere gewöhnlich fast rein erhalten worden bei Auflösen des Ganzen in heissem Weingeist unter Zusatz von etwas Jodwasserstoffsäure, wobei sich alle diese Verbindungen weit leichter als in reinem Weingeist lösen, rorsichtigem Zusatz von Wasser zu der noch heissen Flüssigkeit bis zur bleibenden Trübung, dann von ein wenig Weingeist, und Stehenlassen des Ganzen. Die so erhaltenen scheinen zwar fast rein, jedoch immer ein wenig überschüssigen Jodwasserstoff $\mathrm{zu}$ enthalten.

Die reine Verbindung verliert alles Wasser neben Vitriolöl bei gewöhnlicher Temporatur. Bei kürzerem (einiger Stunden) Erhitzen auf $100^{\circ}$ verliert sie dann nicht an Gewicht, aber bei mehrtägigem Verweilen bei $100^{\circ}$ giebt sie fortwährend kleine Mengen Jod ab.

Meine Analysen der lufttrocknen (nicht neben Vitriolöl getrockneten) Verbindung ergaben folgende Resultate:

$\begin{array}{lrrrrr} & \text { Rechn. } & 1 . & 2 . & 3 \mathrm{a} . & 3 \text { b. } \\ \mathrm{C} & 36,28 & - & 35,88 & - & - \\ \mathrm{H} & 4,20 & - & 4,23 & - & - \\ \mathrm{SO}_{3} & 6,05 & - & 5,96 & 5,94 & - \\ \mathrm{J} & 43,19 & 43,14 & - & - & - \\ \text { Freieres Jod } & 28,79 & 28,80 & 28,80 & 28,90 & 28,00 \\ \mathrm{H}_{2} \mathrm{O} & 2,72 & 2,49 & 2,68 & 2,76 & 2,67\end{array}$

1 war aus der Mutterlauge des 1 ten Cinchonidinperjodidsulfats, 2 aus der Mutterlauge der olivengrünen $\mathrm{Na-}$ deln, 3 als Umbildungsprodukt der olivengrünen Nadeln erhalten. 
Die neben Vitriolöl getrocknete Verbindung ergab bei der Analyse:

Rechn.

$\begin{array}{lr}\mathrm{SO}_{3} & 6,22 \\ \mathrm{~J} & 44,41\end{array}$

\begin{tabular}{|c|c|c|}
\hline \multicolumn{3}{|c|}{ Gefunden. } \\
\hline 6,36 & - & - \\
\hline 44,15 & 44,45 & 44,27 \\
\hline
\end{tabular}

Diese Resultate weichen ganz von denen Herapath's $a b$; er betrachtet nämlich diese und die vorhergehende Verbindung nur als verschierlene Hydrate desselben Atowcomplexes, und in diesem geht, seiner Meinung nach, die das Cinchonidin constituirende Atomgruppe gar nicht ein. Dass aber diese Verbindung unverändertes Cinchonidin enthält, geht daraus hervor, dass ich aus derselben reines Cinchonidin von allen gewöhnlichen Eigenschaften dieses Alkaloids erhalten habe. Es giebt z. B. leicht das charakteristische, schwer lösliche Tartrat, in welchem ich eine Wasserbestimmung ausführte, die 4,55 (Rechn. 4,48) Proc. Wasser lieferte.

Herapath findet nun, dass das luftrockne gelbe Perjodidsulfat neben Vitriolöl 4,71 Proc. an Gewicht verliert und dabei olivenfarbig wird, und dass er dann bei $100^{\circ}$ noch 0,71 Proc. verliert. In einem anderen Versuche - er hat nur diese zwei ausgeführt - findet or zwar, dass die lufttrockne Verbindung bei $100^{\circ}$ 5,86 an Gewicht verliert, meint aber selbst, dass dies Präparat nicht völlig trocken gewesen ist. $\mathrm{Fs}$ ist der so getrocknete olivenfarbene Rückstand, welchen or analysirt hat. In diesem findet er:

\begin{tabular}{lrccc} 
& & & $\begin{array}{c}\text { Rechn. nach moinen } \\
\text { Analysen (8. oben). }\end{array}$ \\
$\mathrm{C}$ & $\mathbf{3 6 , 0 8}$ & $\mathbf{3 5 , 6 9}$ & - & $\mathbf{3 7 , 2 9}$ \\
$\mathrm{H}$ & 4,05 & 4,03 & 4,07 & 4,00 \\
$\mathrm{~N}$ & 2,85 & - & - & 4,35 \\
$\mathrm{SO}_{3}$ & 9,06 & 8,32 & - & 6,22 \\
$\mathrm{~J}$ & 40,50 & 40,41 & - & 44,41 \\
$\mathrm{O}$ als Verlust & $\mathbf{8 , 0 6}$ & - & - & $\mathbf{3 , 7 8}$ \\
\hline
\end{tabular}

Obwohl seine Kohlenstoffbestimmungen nicht eben gut mit meiner Formel stimmen, so ist hier die grosse Schwerverbrennlichkeit dieser Verbindungen wohl zu be- 
riicksichtigen. Die gefundenen Zahlen für Wasserstoff stimmen ausgezeichnet, dagegen die für Jod, Stickstoff und Schwefelsäure gar nicht. Die Abweichungen in den Jodbestimmungen sind, wie ich glaube, im Vorhergehenden hinlänglich beleuchtet. Rücksichtlich der einzigen Stickstoffhestimmung, welche nach Peligot's Methode ausgeführt wurde, so leuchtet so viel ein, dass, da die Verbindung Cinchonidin enthält, was oben nachgewiesen wurde, so muss die Stickstoffbestimmung Herapath's fehlerhaft soin, sofern die Kohlenstoffbestimmungen richtig sind, und umgekehrt. Denn in einer Verbindung von Cinchonidin mit kohlen- und stickstofffreien Stoffen entsprechen

35,89 (Mittel) Proc. Kohlenstoff: 4,18 Stickstoff und 2,85 Proc. Stickstoff:

24,43 Proc. Kohlenstoff.

lis erscheint nun ganz unwahrscheinlich, dass Herapath bei Verbrennung mit Bleichromat in 2 Versuchen iiber 11 Proc. Kohlenstoff zu viel gefundeu haben sollte. Weit natürlicher ist die Annahme, dass die für Stickstoff gefundene Zahl mit einem zufälligen Fehler, einer Fehlrechnung oder dergl., behaftet ist.

In Bezug auf die für Schwefelsäure gefundenen Zahlen, so weichen sie sehr stark sowohl unter einander, als von den naoh meiner Formel zu berechnenden ab, und zwar so stark, dass ich es nicht für möglich halte, die Abwoinhungen durch die oben erwähnten Mängel der Herapath'schen Methode im Allgemeinen zu erklären. Herapath's Präparat hielt unzweifelhaft ïberschüssige Schwofelsäure. Wie sohon angedeutet, sieht er einen Ueberschuss von Schwefelsäure als nothwendige Bedingung der Bildung dieser Verbindung an. Er arbeitete daher in stark schwefelsauren Flüвsigkeiten (auf $1 \mathrm{Mol}$. Cinchonidin 2,25 Mol. Schwefelsäure) und aller Wahrscheinlichkeit nach ist sein Präparat nicht vollständig ausgewaschen worden, vielleicht lässt es sich nicht völlig auswasohen. Mehrere Umstände seheinen hierauf zu deuten. Zuerst der hohe Schwefelsiuregehalt, dann dass nicht nur seine Zahl für das neben Vibriolöl entweichende Wasser zu hoch ist, sondern dass die so getrocknete Substanz, die 9 Tage neben Vitriolöl 
verweilte, noch bei $100^{\circ}$ weiteren Gewichtsverlust erfuhr; endlich dase die reine Verbindung nach moinen Beobachtungen neben Vitriolöl oder bei $100^{\circ}$ gar nicht olivenfarben wird und kaum eine sehr wenig dunklere Nuance annimmt, während die Spuren von freier Schwefelsäure haltende eben jene Farbenänderung erfährt. Alles dies zusammengenommen, scheint mir keine andere Erklärung der von ihm und mir erbaltenen so versehiedenen Resultate möglich, als dass Herapath's Präparat eine geringe Menge freier Schwefelsäure enthielt.

Das reine Perjodidsulfat färbt gar nioht kalten oder kochenden Schwefelkohlenstoff, Aether oder Chloroform. Es löst sich ziemlioh leicht in kochendem Weingeist, und diese Lösung liefert beim Schütteln mit Quecksilbor keine Spur von Quecksilberjodür, sondern ein fast weisses, in kaltem Weingeist schwer lösliches Quecksilberjodiddoppelz, das sich beim Erkalten in seidenglänzenden Rosetten schwach gelblicher, häufig rectangulärer, flacher Nadeln abscheidet. Die siedende weingeistige Lösung des Perjodidsulfats giebt beim Erkalten zuerst messingglanzendes Salz, welches beim Erkalten und Stehen der Flüssigkeit sich sllmählich wieder in die ursprüngliche Verbindung verwsndelt. Wird letztere boi gelinder Wärme und unter Zusatz von ganz wenig Sohwefolsäure in Weingeist gelöst, so ersoheint beim Brkalten zuerst messingglänzendes, dann olivenfarbenes Salz (die folgende Verbindung), in welch' letzteres schliesslioh alles übergeht. Wird dagegen die Verbindung in kochendem Weingeist unter Zusatz von Schwefeleäare und Jod aufgelöst, so krystallisirt beim Erkalten nur messingglänzendes Salz, und zwar ungemein schön.

3 tes Cinchonidinperjodidsulfat. $8 \mathrm{C}_{20} \mathrm{H}_{24} \mathrm{~N}_{2} \mathrm{O}, 5 \mathrm{SH}_{2} \mathrm{O}_{4}, 6 \mathrm{HJ}, \mathrm{J}_{12}, 6 \mathrm{H}_{2} \mathrm{O}$.

Zur Darstellung dieger Verbindung kann man $1 \mathrm{Mol}$. Cinchonidin ( $=8,08 \mathrm{Grm}$.) in $100 \mathrm{Ccm}$. Weingeist von $98^{\circ} \mathrm{T}$. unter Zusetz von $10 \mathrm{Ccm}$. normaler Schwefelshure und 10 Com. normaler Jodwasserstoffsäure auftosen. Wird 
nun die Flïssigkeit mit einer Lösung von 1,5 At. Jod $(=1,905 \mathrm{Grm}$.) in $50 \mathrm{Cem}$. Weingeist so vermischt, dass das Gemenge nach der Mischung eine Temperatur von etwa $55^{\circ}$ enthält, so scheiden sich sebr bald mehrere $\mathrm{Cm}$. lange, dünne olivengrüne, halb metallglänzende Nadeln der gesuchten Verbindung ab. Diese müssen vor dem vollständigen Erkalten der Flüssigkeit (bei etwa 30") abfiltrirt werden, sonst mischt sich die vorhergehende Verbindung bei. Das Filtrat liefert nur letztere. Verfährt man wie obeu, wendet aber auf $1 \mathrm{Mol}$. Cinchonidin nur 1 At. Jod an, so erhält man bei einer Temperatur von etwa $40^{\circ}$ und Steken nur die olivengrüne Verbindung, und selbst wenn diese 14 Tage unter der Mutterlauge hingestellt wird, findet man sehr wenig gelbe Nadeln beigemischt. Das 3 te Cinchonidinperjodidsulfat, welches von Herapath nicht erwähnt wird, wirkt sehr kräftig polarisirend: \| der Axe fast farblos, + dunkelolivenbrann, fast undurchsichtig. Bei 100 bis $110^{\circ}$ verliert das Salz alles Wasser. Aus der kochenden weingeistigen Lösung der reinen Verbindung krystallisiren zuerst grüne Blätter, die an messingglänzendes Salz sehr erinnern, jedoob mehr gräulich sind. Aber diese werden schnell, schon vor völligem Erkalten, zu den olivengrünen Nadeln. Sie scheinen dabei eine $Z$ wischenstufe durchzulaufen, indem anscheinend zuerst die folgende Verbindung gebildet wird. Genauer habe ich die Sache nicht untersuchen künnen, weil der Uebergang so allmählich geschieht, dass die Flüssigkeit zugleich alle 3 Verbindungen enthalten kann.

Analysen der neben Vitriolöl getrockneten Verbindung:

\begin{tabular}{|c|c|c|c|c|c|c|}
\hline & Reohn. & a. & a. & b. & c. & o. \\
\hline $\mathrm{SO}_{3}$ & 7,47 & 7,67 & - & 7,73 & - & - \\
\hline J & 42,80 & 42,83 & - & 42,61 & - & - \\
\hline Freieres Jod & 28,46 & 28,20 & 28,50 & 28,20 & 28,20 & 28,20 \\
\hline $\mathrm{H}_{\mathbf{2}} \mathrm{O}$ & 2,02 & - & - & 2,32 & 2,06 & - \\
\hline
\end{tabular}

a war aus $1 \mathrm{Mol}$. Cinchonidin und 1,5 At. Jod, b und c aus 1 At. Cinchonidin und 1 At. Jod dargestellt.

Die Verbindung färbt nicht Schwefelkohlenstoff, Aether 
oder Chloroform weder in der Kälte, noch beim Kochen. Quecksilber liefert mil der heissen weingeistigen Lösung kein Jodür, sondern ein Jodiddoppelealz, das beim Erkalten der weingeigtigen Lösung sich als gelblich weisse, kleine Rosetten häufig rectangulärer, flachèr doppelbrechender Nadeln abeetzt. Das Perjodidsalfat wird in saurer, besonders jodwasserstoffseurer Flüssigkeit schon in der Kälte leicht zu der vorhergehenden Verbindung umgebildet.

$$
\begin{aligned}
& 4 \text { tes Cinchonidinperjodidsulfat. } \\
& 2 \mathrm{C}_{20} \mathrm{H}_{24} \mathrm{~N}_{2} \mathrm{O}, \mathrm{SH}_{2} \mathrm{O}_{4}, \mathrm{HJ}, \mathrm{J}_{4}, \mathrm{H}_{2} \mathrm{O} \text {. }
\end{aligned}
$$

Diese, von Herapath auch nicht erwähnte Verbindung, die dem unten beschriebenen 2 ten Cinchonidinperjodidseleniat entspricht, wird leicht und sicher aus der berechneten Menge der Bestandtheile in siedender weingeistiger Lösung erhalten (in Allem etwr $150 \mathrm{Ccm}$. Weingeist von $93^{\circ}$ T. uf $3,08 \mathrm{Grm}$. Cinchonidin). Bei langsamem Erkalten kann sich zwar ein wenig messingglänzendes Salz bilden, und immer nur wenig, aber bei weiterem Erkalten wird Alles zu rothbrannen, oder bèi sehr langsamem Erkalten dunkelbraunen Rosetten kurzer, stark glänzender, schöner Nadeln, dem entspreobenden Seleniat zum Verwechseln ähnlich, und die unverändert umkrystallisirt werden können. Die Verbindung polarisirt das Licht: If der Axe braungelb, + dunkelbraun bis undurchsichtig. Sie verliert kein Wasser, sondern eine bedeutende Menge Jod bei $100^{\circ}$. In einem Versuche gab $1 \mathrm{Grm}$. in 6 Stunden bei $100^{\circ} 0,0842$ ab und verlor auch bei fortgesetzter Erwärmung noch an Gewicht (für jedo Stunde etwa $1 / 2$ Mgrm.). Bei $110^{\circ}$ giebt die Verbindung alles Wasser ab.

Die Analyse der neben Vitriolül getrackneten Verbin-

\begin{tabular}{|c|c|c|c|}
\hline $\mathrm{SO}_{3}$ & $\begin{array}{c}\text { Reobn. } \\
5,85\end{array}$ & $\begin{array}{c}\text { a. } \\
6,04\end{array}$ & b. \\
\hline$J$ & 46,42 & 46,49 & - \\
\hline Freieres Jod & 37,14 & 37,20 & 37,40 \\
\hline $\mathrm{H}_{2} \mathrm{O}$ & 1,32 & - & 1,32 \\
\hline
\end{tabular}
dung ergab: 
382 Jorgensen: Ueber den sogenannten Herapathit

Die Verbindung färbt weder kalten, noch kochenden Schwefelkohlenstoff, Aether oder Chloroform. Quecksilber giebt beim Schütteln mit der heissen weingeistigen Lösung Jodür und ein Jodiddoppelsalz, dem von der vorhergehenden Verbindung gebildeten sehr ähnlich. Vertheilt man das Perjodidsulfat in Weingeist und setzt ein wenig verdünnte Schwefelsäure und verdünnte Jodwasserstoffsäure hinzu, so bildet sich schon in der Kälte sogleich das messingglänzende Salz, welches, beim Kochen gelöst, bei langsamem Erkalten sehr hübsch krystallisirt. Löst man 4 tes Cinchonidinperjodidsulfat in siedendem Weingeist unter Zusatz von etwas Jodwasserstoffsäure, wobei die Lösung sehr leicht vor sich geht, so scheiden sich beim Verdünnen der heissen Lösung mit Wasser und Stehenlassen, schöne gelbbraune Nadeln des 2 ten Cinchonidinperjodidsulfats $\mathbf{a b}$.

Die auf $100^{\circ}$ erhitzt gewesene Verbindung, die, wie schon oben bemerkt, hierbei viel Jod verloren, schmilzt theilweise beim Kochen mit Weingeist. Ner bei Weitem grössere Theil löst sich jedoch und scheidet sich beim Erkalten theils als rothbraunes Pulver, grösstentheils aber als olivengrüne Nadeln vom 3 ten Cinchonidinperjodidsulfat sus.

Von diesen 4 Verbindungen scheinen die 2 te und 3 te, die 1 te und 4 te in näherem Zusammenhang zu stehen. Bezeichnet man durch $\mathrm{M}$ ein $\mathrm{Mol}$. neutralen schwefelsauren Cinchonidins $=\mathrm{C}_{20} \mathrm{H}_{24} \mathrm{~N}_{2} \mathrm{O}, \mathrm{H}$. O. $\mathrm{SO}_{2}$. O. H, $\mathrm{C}_{20} \mathrm{H}_{24} \mathrm{~N}_{2} \mathrm{O}$, so scheint 2 tes Cinchonidinperjodidsulfat am einfachsten so ausgedrückt werden zu können:

$$
\left\{\begin{array}{l}
\mathrm{J}_{2}, \mathrm{~J}, \mathrm{H} \mathrm{M} \mathrm{H}, \mathrm{J}, \mathrm{J}_{2} \\
\mathrm{~J}_{2}, \mathrm{~J}, \mathrm{H} \mathrm{M}
\end{array}\right.
$$

Im 3 ten scheinen solche 2 Moleküle durch 1 Schwefelsäuremolekül verbunclen za sein: 


$$
\begin{aligned}
& \left\{\begin{array}{l}
J_{2}, \text { J.HMH.J.J } \\
J_{2} \cdot \text { J.HMH.O. }
\end{array}\right. \\
& \left\{\begin{array}{l}
J_{2} . J . H . M H . O . J O_{2} \\
J_{2} . J . H M H . J . J_{2}
\end{array}\right.
\end{aligned}
$$

So erklärt sich leicht der leichte Uebergang von einem zu anderen. Zwischen 1 ter und 4 ter Verbindung ist in Vorhergehendem kein Zusammenhang bestimmt nachgewiesen; dass aber ein solcher sich findet, und zwar ein sehr enger, zeigt die dem 1 ten Cinchonidinperjodidsulfat entsprechende Selensüureverbindung, die weiter unten beschrieben werden soll. Dieselbe zersetzt sich nämlich mit sehwachem Weingeist so, dass $2 \mathrm{Mol}$. Jodwasserstoff und 3 Mol. Selensäure aus dem Molekül heraustreten, wobei dasselbe in $6 \mathrm{Mol}$. des dem 4 ten Cinchonidinperjodidsulfat entsprechenden Seleniate zufällt. Diese Zersetzung lässt sich durch folgende Formel am einfachsten ausdrücken, wo $M^{\prime} 1$ Mol. neutrales Cinchonidinseleniat bedeutet:

$$
\begin{aligned}
& \left.\begin{array}{l:l}
\mathrm{J}_{4} . J . H M^{\prime} & \mathrm{H} . \mathrm{O} \\
\mathrm{J}_{4} . \mathrm{J} . \mathrm{H} \mathrm{M}^{\prime}: \mathrm{H} . \mathrm{O}
\end{array}\right\} \mathrm{Se} \mathrm{O}_{2} \\
& \mathrm{~J}_{2} \text {. J . H } \\
& \left.\begin{array}{l}
\mathrm{J}_{2} \cdot \mathrm{J}^{\mathrm{H}}: \mathrm{H}^{\prime}: \mathrm{H} . \mathrm{O} \\
\mathrm{J}_{2} \cdot \mathrm{J} . \mathrm{H}_{\mathrm{M}^{\prime}} \mathrm{H} . \mathrm{O}
\end{array}\right\} \mathrm{SeO}_{2}=6 \mathrm{~J}_{4} \cdot \mathrm{J} . \mathrm{HM}^{\prime}+2 \mathrm{HJ}+3 \mathrm{SeH}_{2} \mathrm{O}_{4} \\
& \mathrm{~J}_{2} \text {.J.H } \\
& \left.\begin{array}{l:l}
\mathrm{J}_{4} \cdot \text { J } . \mathrm{H}^{\prime} & \mathrm{H} . \mathrm{O} \\
\mathrm{J}_{4} \cdot \text { J } . \mathrm{H} \mathrm{M}^{\prime}: \mathrm{H} . \mathrm{O}
\end{array}\right\} \mathrm{Se} \mathrm{O}_{2}
\end{aligned}
$$

Wenn die Schwefelsäureverbindung, wenigstens so weit ich beobachtet habe (vergl. oben), kein solches Verhalten zeigt, so kann das darin seinen Grund haben, dass die Selensäure in ihren Alkaloidverbindungen loser als die Schwefelsüure gebunden ist. Wenigstens deutet der Umstand, dass sich nach Oppenheim ${ }^{1}$ ) die Tellursäure weder mit Morphin, Chinin, noch Cinchonin verbindet, darauf hin. Wenn die 2 Mol. Jodwasserstoff im messingglänzenden Salz loser gebunden, so hat dies wohl

1) Dies Journ. 71, 266. 
384 Jorgensen: Ueber den sogenannten Herapathit

einfach seinen Grand darin, dass sie als übersaures Salz zugegen sind.

H. Perjodidsulfate monovalenter Basen.

Auch Anilin und Toluidin bilden àhnliche Verbindungen, obwohl von weit weniger stabiler Natur, und von ganz eigenthümlicher, schwer zu deutender Zusammensetzung, wenn man nicht die leichte Zuflucht zu molekularen Verbindungen nimmt.

Anilinperjodidsulfat. $\quad 6 \mathrm{C}_{6} \mathrm{H}_{7} \mathrm{~N}, 3 \mathrm{SH}_{2} \mathrm{O}_{4}, \mathrm{HJ}, \mathrm{J}_{4}$.

Werden $6 \mathrm{Mol}$. Anilin (5,58 Grm.) in 6 bis $7 \mathrm{Mol}$. normaler Schwefelsäure und $200 \mathrm{Ccm}$. Wasser gelöst, das Gemisch auf 30 bis $40^{\circ}$ erwärmt und eine ebenfalls schwach erwärmte Lösung von 4 At. Jod in $1 \mathrm{Mol}$. normalen Jodwasserstoff's und $50 \mathrm{Ccm}$. Weingeist von $93^{\circ} \mathrm{T}$, zugesetzt, so wird bald ein glänzender, herapathitähnlịcher, krystallinischer Niederschlag dieser Verbindung erbalten. Er ist mit schwachem Weingeist (etwa 40 $0^{\circ}$.) zu waschen (Wasser zersetzt theilweise), nach dem (schnellen) Auswaschen sofort zwischen Papier zu pressen und veben Vitriolöl im Dunkeln za trocknen. Hierbei behalten die Krystalle den Glanz und rerlieren, so getrocknet, kaum an Gewioht durch kürzeres Erwärmen auf $100^{\circ}$. Sie sind in Dunkelu aufzubewahren, da sie, obwohl langsam, im Lichte und an der Luft theilweise zersetzt werden.

In Masse ist die Verbindung metallgrün, fast genau von der Farbennuance des Herapathits. Sie bildet rhombische Blätter, die nicht oder wenigstens äusserst schwach das Licht polarisiren. In durchfallendem Licht sind aie schön und bell rothbraun, von einer eigenthümlichen, sehr charakteristischen Nuance, die etwas in's Violett neigt. Die Blätter sind immer von $\overline{\mathrm{P}} \infty . \propto \overline{\mathrm{P}} \infty$ begrenzt, $\overline{\mathrm{P}} \infty: \overline{\mathrm{P}} \infty$ gef. $=99,75^{\circ}$ bis $100^{\circ}$ (20 Messungen an $6 \mathrm{Krystallen}$ verschiedener Darstellungen); $\overline{\mathrm{P}} \infty: \infty \overline{\mathrm{P}} \infty 0=190^{\circ}$ bis $190,5^{\circ}$ (5 Messungen an 3 Krystallen). 
Analysen der neben Vitriolöl getrockneten Verbindung:

$\begin{array}{lrcccc} & \text { Rochn. } & \text { a. } & \text { b. } & \text { c. } & \text { d. } \\ \text { C } & 29,03 & - & - & - & 28,97 \\ \mathrm{H} & 3,29 & - & - & - & 3,28 \\ \mathrm{SO}_{3} & 16,19 & 16,33 & 16,32 & - & 16,01 \\ \text { J } & 42,60 & 42,36 & - & 42,65 & 42,53 \\ \text { Freieres Jod1) } & 34,14 & - & - & 33,20 & 33,90\end{array}$

Dass die Verbindung unverändertes Anilin enthält, wies ich auf folgende Weise nach. Das gepulverte, in Wasser vertheilte Salz wurde durch Schwefelwasserstoff zersetzt, das Filtrat von dem (grauen) Schwefel bedeutend eingeengt und mit Natron versetzt, die milchige Flüssigkeit mit Aether ausgeschüttelt, der anilinhaltige Aether mit Wasser, dann mit verdünnter Schwefelsäure geschüttelt. Dabei schied sich krystallisirtes schwefelsaures Anilin ab, welches sich jedoch durch Zusatz von mehr Wasser und Schütteln löste. Diese Lösung mechanisch vom Aether getrennt, gab, mit Natron neutralisirt, die bekannten Anilinreactionen mit Chloruatron und mit weingeistigem Natron und Chloroform, und (ohne Neutralisation) wieder die ursprüngliche Verbindung.

Reines Wasser löst die Verbindung in nicht geringer Menge. Die Lösung ist braun, riecht nach Jod und trocknet neben Vitriolöl oder bei freiwilligem Verdunsten zu einer dünnen Schicht ein, der ähnlich, welche die Rosanilinsalze unter denselben Umständen lieforn. Sie zeigt sich nämlich metallisch grün in reflectirtem, roth in durchfallendem Licht, jedoch von ganz anderer Nuan se als die Rosanilinsalze. Beim . Kochen mit Wasser entwickeln sich Joddämpfe, die Verbindung scheint zu schmelzen, schliesslich

1) $\mathrm{Zu}$ dieser Bestinmung wurden 0,5 bis $0,8 \mathrm{Grm}$. der fein gepulverten Verbindung mit ca. $60 \mathrm{Ccm}$. kaltem Weingeist von $930 \mathrm{~T}$. ïbergossen, wobei der grössere Theil sich löste. Die Entfärbung geschah leicht und die Lösung wurde bald vollständig durch Zusatz ron Natriunhyposultit. Zwar warde die Lösung nicht farblos, wegen Einwirkung von Licht und Luft, sondern gräulich. Der Uelergang in's Gelbe war jedoch bei 1 Tropfen $1 / 10$ normaler Jodlösung kennbar.

Journal f. prakt. Ohemie [2] Bd. 14. 
386 Jorgensen: Ueber den sogenannten Herapathit etc.

löst sich alles fast farblos auf. Auch Weingeist von $93^{\circ}$ T. löst ziemlich reichlich, absoluter schwieriger. Aether wird schwach bräunlich gefärbt, Chloroform und Schwefelkohlenstoff färben sich nicht in der Kälte und auch bein Kochen ziemlich schwach. - Beim Sehütteln mit Quecksilber und heissem Weingeist bildet sich viel Quecksilberjodür. Das Filtrat hält ein Quecksilberjodiddoppelsalz, das beim Stehen sich als mikroskopische, farblose, kurze, äusserst dïnne verfilate Nadeln abscheidet.

Toluidinperjodidsulfat. $6 \mathrm{C}_{7} \mathrm{H}_{9} \mathrm{~N}, 3 \mathrm{SH}_{2} \mathrm{O}_{4}, \mathrm{HJ}, \mathrm{J}_{4}$.

Das Paratoluidin giebt ganz wie Anilin ein Perjodidsulfat, welches der Anilinverbindung sehr ähnlich ist, selten sber regelmässig krystallisirt, sondern in zerrissenen und gebrochenen Blättern auftritt. Es verändert sich weit schneller, als das Anilinsalz in Licht und Luft. Ich habe ein paar Bestimmungen ausgeführt, die jedoch zeigen, dass das Salz der Anilinverbindung entspricht. Die Analysen beziehen sich auf in trocknem und dunklem Vacuum getrocknete Substanz.

$\begin{array}{lcc} & \text { Rechn. } & \text { Gefunden. } \\ \mathrm{J} & \mathbf{4 0 , 3 9} & 40,70 \\ \mathrm{SO}_{3} & 15,27 & 15,32\end{array}$

Versuche, die Menge des freien Jods zu bestimmen, gaben bei der grossen Veränderlichkeit der Verbindung ungenügende Resultate (zwischen 29 und 34 Proc., Rechn. 31,7). Bei sehr langem Erwärmen auf $100^{\circ}$ verliert die Verbindung etwa 30 Proc. an Gewicht, oxydirt sich aber gleichzeitig theilweise.

Es giebt mehrere hierher gehörige Verbindungen. So hildet Anilin eine ganz ähnliche mit Salpetersäure, statt Schwefelsäure. Alle diese sind aber so leicht zersetzbar, dass es mir nicht gelang, sie in zur Analyse verwendbarem Zustande zu erhalten. Toluidinperjodidphosphat zeichnet sich übrigens durch seine eigenthümlichen optischen Verhältnisse aus. In Masse ist es dem Anilinperjodidsulfat sehr ähnlich, unter dem Mikroskop erscheint es aber als 
elliptisehe Blätter, die Zwillinge und Vierlinge zu sein scheinen. Erstere zeigen das optische Verhalten: $\|$ der Längsaxe dunkelbraun, fast undurchsichtig, + hellbraun bis gelbbraun, $x$ die eine Hälfte (nach der langen Axe getheilt) dunkel, die andere hell. Letztere zeigen Folgendes: II der langen Axe hell blassgrün, + rothviolett; steht aber der Hauptsehnitt des Nicols in einer Zwischenstellung, so erseheint die Krystallamelle in 4 Quadranten getheilt, die zwei gegenüberliegenden sind blassroth, die zwei zwischenliegenden rothviolett; steht, der Hauptschnitt des Nicols in der auf letzteren senkrechten Stellung, so sind die Farben umgetauscht.

Kopenhagen, Polytechnische Lehranstalt, October 1876.

(Wird fortgesetzt.)

\title{
Zur Ultramarinfrage;
}

\author{
von \\ W. Stein.
}

Die werthvollsten Aufschlüsse uiber den Ultramarin verdanken wir den Arbeiten R. Hoffmann's, bei dem sich gründliche Kenntniss der praktischen Verhältnisse mit scharfer Beobachtungsgabe und besonnenem wissenschaftlichem Urtheile vereint findet. Wenn es durch diese Arbeiten noch nicht gelungen ist, die Frage zu entscheiden, in welcher metallischen Verbindung der Schwefel im Ultramarin sich befindet, so wird man wohl annehmen dürfen, dass dieselbe auf diesem Wege übeshaupt nicht $\mathrm{zu}$ lösen sei. Einen weiteren Beweis hierfür liefern die „praktisch-theoretischen Studien" der Herren Dollfuss und Goppelsröder (Dingl: Journ. 1876, S. 337 u. 431 ff.), zu denen ich einige berichtigende Bemerkungen zu machen mich genöthigt sehe, da die von mir zu dieser Frage ge- 